\title{
Signatures of Majorana Fermions in Hybrid Superconductor-Topological Insulator Devices
}

\author{
J. R. Williams, ${ }^{1}$ A. J. Bestwick, ${ }^{1}$ P. Gallagher,${ }^{1}$ Seung Sae Hong, ${ }^{2}$ Y. Cui,,${ }^{3,4}$ \\ Andrew S. Bleich, ${ }^{5}$ J. G. Analytis,,${ }^{2,4}$ I. R. Fisher, ${ }^{2,4}$ and D. Goldhaber-Gordon ${ }^{1}$ \\ ${ }^{1}$ Department of Physics, Stanford University, Stanford, CA 94305, USA \\ ${ }^{2}$ Department of Applied Physics, Stanford University, Stanford, CA 94305, USA \\ ${ }^{3}$ Department of Material Science, Stanford University, Stanford, CA 94305, USA \\ ${ }^{4}$ Stanford Institute for Materials and Energy Sciences, \\ SLAC National Accelerator Laboratory, Menlo Park, California 94025, USA. \\ ${ }^{5}$ Geballe Laboratory for Advanced Materials, Stanford University, Stanford, CA 94305, USA
}

(Dated: February 22, 2012)

\begin{abstract}
The ability to measure and manipulate complex particles in the solid state is a cornerstone of modern condensed-matter physics. Typical excitations of a sea of electrons, called quasiparticles, have properties similar to those of free electrons. However, in recent years exotic excitations with very different properties have been created in designer quantum materials, including Dirac fermions in graphene ${ }^{1}$ and fractionally-charged quasiparticles in fractional quantum Hall systems ${ }^{2}$. Here we report signatures of a new quasiparticle - the Majorana fermion - in Josephson junctions consisting of two superconducting leads coupled through a three-dimensional topological insulator ${ }^{3}$. We observe two striking departures from the common transport properties of Josephson junctions: a characteristic energy that scales inversely with the width of the junction, and a low characteristic magnetic field for suppressing supercurrent. To explain these effects, we propose a phenomenological model in which a one-dimensional wire of Majorana fermions is present along the width of the junction, similar to a theoretical prediction by $\mathrm{Fu}$ and $\mathrm{Kane}^{4}$. These results present an opening into the investigation of Majorana fermions in the solid state and their exotic properties, including non-Abelian statistics ${ }^{5}$, a suggested basis for fault-tolerant quantum computation ${ }^{6}$.
\end{abstract}

The Majorana fermion, a charge-neutral particle that is its own antiparticle, was proposed theoretically almost 75 years ago $^{7}$. In the original context of high-energy physics, neutrinos have been proposed to be Majorana fermions, a designation that could help explain the domination of matter over antimatter in our universe ${ }^{8}$. Separately, electronic excitations in certain condensed matter systems have recently been predicted to act as Majorana fermions ${ }^{7}$. One such system is a three-dimensional topological insulator (TI) where superconducting correlations between particles are introduced, producing a "topological superconductor" with properties similar to a $p+i p$ superconductor ${ }^{3}$. Such superconducting correlations can be induced by doping a TI with $\mathrm{Cu}$, where deviations from conventional superconductivity have been seen ${ }^{9}$. Another way to create a topological superconductor is via the proximity effect, wherein a conventional superconductor is placed in contact with a $\mathrm{TI}^{10}$. When two superconductors are connected by a TI, the TI "weak link" superconducts due to its proximity to the superconducting leads. This produces a Josephson junction (JJ) but with several important distinctions compared to a conventional JJ, where the weak link is typically an ordinary metal or insulator. Fu and Kane have predicted [4] a one-dimensional (1D) mode of Majorana fermions at the interface between a conventional superconductor and a superconducting topological surface state. Hence, JJs formed with a TI weak link are expected to have two $1 \mathrm{D}$ modes at the two superconductor-TI interfaces (arrows in Fig. 1a), which fuse to form a 1D wire of Majo- rana fermions (shown in purple in Fig. 1a) running along the width of the device ${ }^{4}$. The energy spectrum of these Majorana fermions is characterized by states within the superconducting gap, which cross at zero energy when the phase difference $\varphi$ between the two superconducting leads is $\pi$ (solid purple line, Fig. 1b).

To probe this exotic state, recent experiments have investigated transport in TI JJs, finding good agreement with conventional JJ behavior ${ }^{11-15}$. Two characteristic properties are typically reported for JJs. The first is the product $I_{C} R_{N}$, where $I_{C}$ is the critical current and $R_{N}$ is the normal state resistance. $I_{C} R_{N}$ should be of order $\Delta / e$ (where $\Delta$ is the superconducting gap of the leads and $e$ is the charge of the electron) and independent of device geometry ${ }^{16}$. Two experiments ${ }^{11,13}$ have noted a smaller-than-expected value for the product $I_{C} R_{N}$ in TI JJs. The second characteristic property is the "Fraunhofer-like" magnetic diffraction pattern, i.e. the decaying, oscillatory response of the supercurrent to the magnetic field $B$, applied perpendicular to the flow of the supercurrent. The first minimum in $I_{C}$ should occur at $B=B_{C}$, when one quantum of flux $\Phi_{0}=h / 2 e$ (where $h$ is Planck's constant) is passed through the area of the device, and recent reports on in TI JJs ${ }^{14,15}$ match this expectation.

The main experimental results of this paper are a small value of $I_{C} R_{N}$ that scales with $1 / W$, where $W$ is the width of the JJ, and a value of $B_{C}$ that is $\sim 5$ times smaller than that expected from the device area. Neither of these results is predicted or previously seen for 


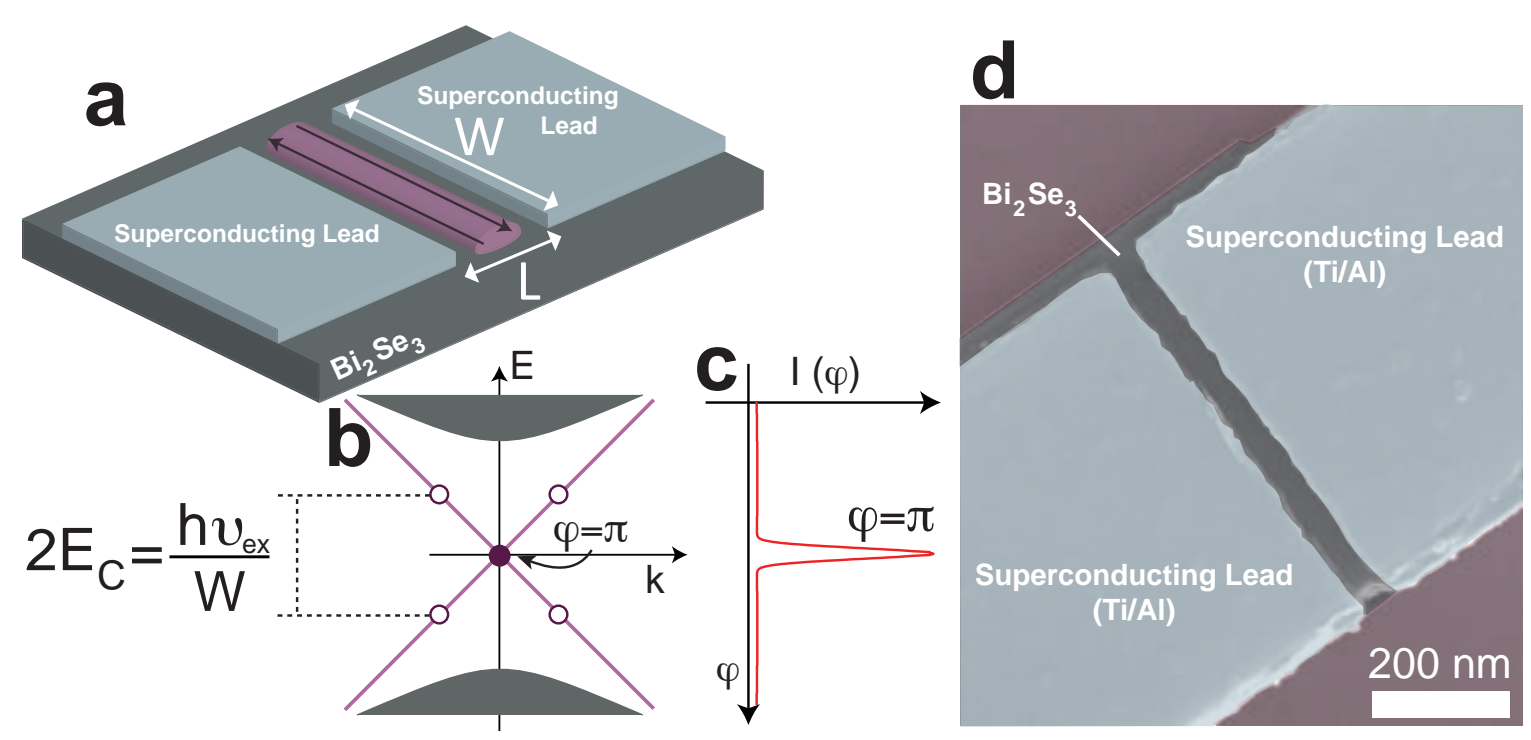

FIG. 1: Phenomenological model for Majorana fermions in topological insulator Josephson junction. a, Schematic of a topological insulator Josephson junction. Two superconducting leads are patterned on top of $\mathrm{Bi}_{2} \mathrm{Se}_{3}$ forming a junction with length $L$ and width $W$. Along the width of the device, a one-dimensional wire of Majorana fermions results (purple). b, Energy levels near $\varphi=\pi$ before momentum quantization along $W$ (purple line) and after, where the topological state remains at $E=0$ (solid purple circle) and the first quantized energy level at the value $E_{C}$ (empty purple circles). c, Current-phase relation resulting from momentum quantization, producing an anomalous peak at $\varphi=\pi$. d, Scanning-electron micrograph of a device similar to the ones measured in this Letter.

conventional JJs nor TI JJs. To explain these experimental observations, we propose a two-fold phenomenological extension to the model in Ref 4 . First we take into account confinement along the 1D Majorana wire, quantizing its energy levels at multiples of $E_{C}=h \nu_{e x} / 2 l$, with $\nu_{e x}$ the velocity of the carriers in the wire and $l$ the length of the wire. In the present devices, the length of the wire is the width $W$ of the JJ, hence $E_{C}=h \nu_{e x} / 2 W$. The effect of this quantization on the energy levels is shown in Fig. 1b. If the $E=0$ state (Fig. 1b, solid purple dot) is topological in nature, i.e. it is a neutral Majorana mode, such confinement should not affect its existence nor change its energy from zero. The continuum of energy levels at $E \neq 0$, not protected from perturbations, are quantized in multiples of $E_{C}$ (Fig. 1b, empty purple dots where only the first non-zero energy modes are shown for clarity). The second extension of Ref. 4 is to postulate that only the topological $E=0$ Majorana state carries supercurrent, while for $E \neq 0$ (separated from the topological state by $E_{C}$ ) the transfer of carriers from one lead to the other is dissipative. This results in an $I_{C} R_{N}$ set by the energy scale of confined modes along the width of the junction, rather than by $\Delta / e: I_{C} R_{N} \propto E_{C} / e=h \nu_{e x} / 2 e W$. This also has an effect on the current-phase relation, which determines the supercurrent through the device as a function of $\varphi$. The supercurrent-carrying $E=0$ state occurs at $\varphi=\pi$, producing a sharp peak in the current-phase relation at $\varphi=\pi$ (Fig. 1c), in contrast with the established sinusoidal current-phase relation for conventional $\mathrm{JJs}^{16}$, or a different-period sinusoid predicted for TI JJs ${ }^{17}$.
As described above, the result of confinement is to separate in energy the $E=01 \mathrm{D}$ modes (neutral Majorana modes) from the $E \neq 0$ (charged) $1 \mathrm{D}$ modes. A 1D mode can carry a supercurrent, but the critical current is strongly suppressed by interactions between charges ${ }^{18,19}$. Thus a neutral mode can carry a larger supercurrent than a charged mode. In our experiment the lowest-energy charged modes are accessed when the current through the neutral mode is $1 \mu \mathrm{A}$. We estimate that the charged modes cannot carry this much supercurrent. Hence the charged modes in our devices act as resistors carrying current but not supercurrent. When the charged modes become energetically accessible (i.e. for energies $\geq E_{C}$ ) they shunt the junction, causing the JJ to undergo a superconducting to metallic phase transition ${ }^{20}$, which takes places because the 1D-charged-mode shunt resistance is greater than $h / 4 e^{2}{ }^{21}$. In this model, any additional supercurrent through the bulk (which might be expected in existing TIs, given the finite bulk conductivity) also ceases when the shunt resistance of the surface become energetically accessible.

To investigate the properties of JJs with TI weak links, $\mathrm{Bi}_{2} \mathrm{Se}_{3}$ crystals were synthesized either via a slow cooling of a binary melt of $\mathrm{Bi}$ and $\mathrm{Se}^{22}$ or by vapor-liquid-solid (VLS) growth ${ }^{23}$ and flakes of thicknesses between 50 and $100 \mathrm{~nm}$ were obtained from these crystals via mechanical exfoliation ${ }^{1}$. Junctions of lengths $L$ between 20 and $80 \mathrm{~nm}$ and widths $W$ between 0.5 and $3.2 \mu \mathrm{m}$ were fabricated via electron-beam lithography, followed by deposition of $\mathrm{Ti} / \mathrm{Al}$ electrical leads (see the Supp. Info for details of the sample fabrication). A scanning electron 


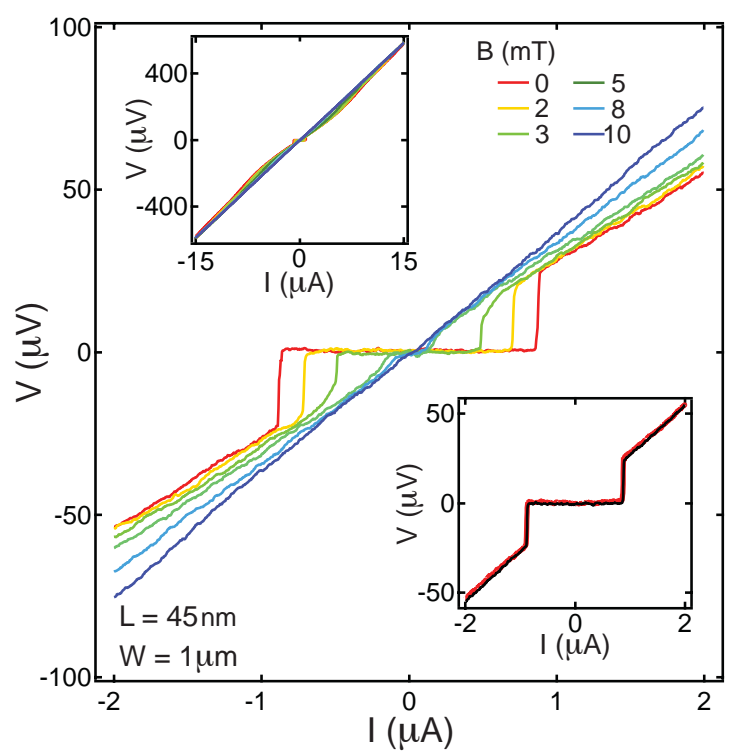

FIG. 2: $\boldsymbol{I}-\boldsymbol{V}$ response of a topological insulator Josephson junction. (main) $V$ vs. $I$ for a devices of dimensions $(L, W)=(45 \mathrm{~nm}, 1 \mu \mathrm{m})$ for $B=0,2,3,5,8,10 \mathrm{mT}$ and at a temperature of $12 \mathrm{mK}$. At $B=0, I_{C}$ is $850 \mathrm{nA}$, which is reduced upon increasing $B$. For this device, the product $I_{C} R_{N}=30.6 \mu \mathrm{V}$, much lower than theoretically expected for conventional JJs. (upper-left inset) $I-V$ curves overlap for all values of $B$ at $V \geq 2 \Delta / \mathrm{e} \sim 300 \mu \mathrm{V}$. (lower-right inset) Sweeps up (red) and down (black) in $I$ show little hysteresis, indicating that the junction is in the overdamped regime.

micrograph of a completed device is shown in Fig. 1d. The DC response for a $(L, W)=(45 \mathrm{~nm}, 1 \mu \mathrm{m})$ junction at a temperature of $12 \mathrm{mK}$ is shown in Fig. 2, where the DC voltage $(V)$ is plotted as a function of the applied DC current $(I)$. At $B=0$, a typical DC Josephson response is observed (red curve): for $I \leq I_{C}=850 \mathrm{nA}, V=0$ and a supercurrent flows. Applying $B$ perpendicular to the top surface of the $\mathrm{Bi}_{2} \mathrm{Se}_{3}$ reduces $I_{C}$ until $B=10 \mathrm{mT}$ when the superconducting leads are driven normal and the $\mathrm{I}-\mathrm{V}$ curve becomes linear. For $I>I_{C}$, there is an excess current due to Cooper pairs leaking into a lowbarrier junction ${ }^{24}$; this excess decreases with $B$. For $V \geq$ $2 \Delta / \mathrm{e} \sim 300 \mu \mathrm{V}$, all curves fall on top of each other (upper left inset of Fig. 2) for all values of $B$. Absence of hysteresis (lower right inset of Fig. 2), indicates that the junction is overdamped, consistent with calculations (Supp Info). $R_{N}$ for this device is $35 \Omega$ and $I_{C} R_{N}=30.6 \mu \mathrm{V}$. Theoreretical expectations ${ }^{25}$ for diffusive and ballistic weak links predict $I_{C} R_{N}$ to be $281 \mu \mathrm{V}$ and $427 \mu \mathrm{V}$, respectively, an order of magnitude higher than our measurements. As a control experiment, a device fabricated similarly to the TI JJs, except with a $75 \mathrm{~nm}$-thick graphite weak link, has $I_{C} R_{N}=244 \mu \mathrm{V}$ (Supp. Info.), much closer to theoretical predictions. This suggests that something in the sample rather than the measurement setup reduces the values of $I_{C} R_{N}$.

Further insight into the nature of transport in TI JJs is
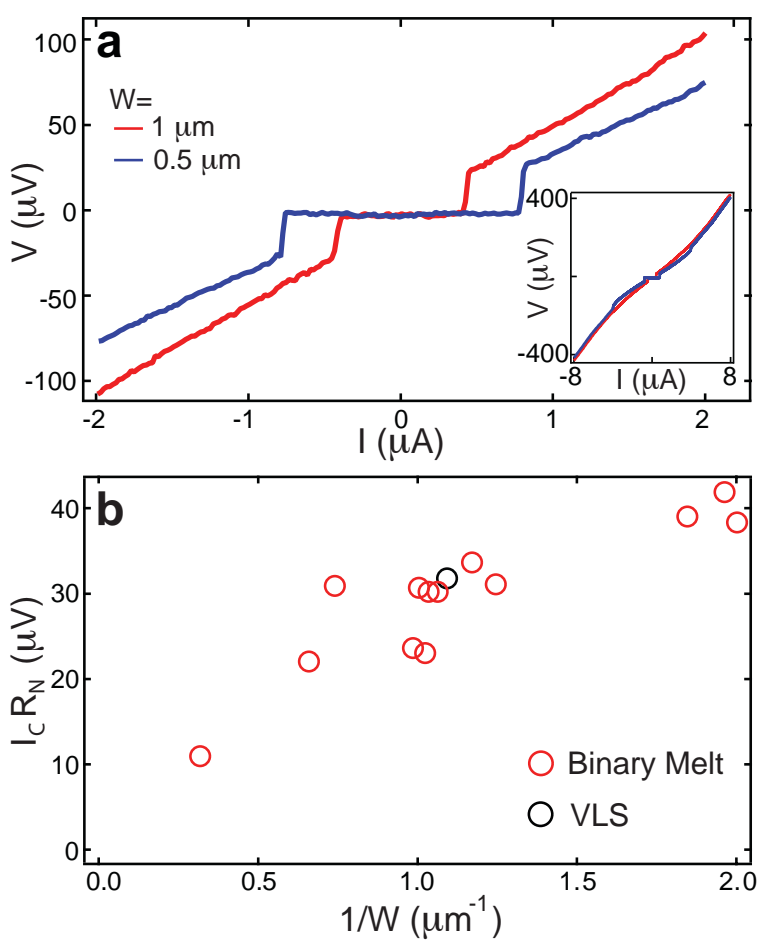

FIG. 3: $1 / \boldsymbol{W}$ dependence of $\boldsymbol{I}_{C} \boldsymbol{R}_{N}$. a, A comparison of two devices with similar $R_{N}$ (56.1 and $51.5 \Omega$ ) and different widths $W, 0.5$ and $1 \mu \mathrm{m}$. The device with $W=0.5 \mu \mathrm{m}$ exhibits a larger $I_{C}$, in contrast to conventional JJs, where similar resistances lead to similar values of $I_{C} . \quad \mathbf{b}, I_{C} R_{N}$ vs. $1 / W$ for all 14 devices (synthesized via two methods: a binary melt and VLS) showing the general trend of $I_{C} R_{N} \propto 1 / W$. Using the phenomenological model of Fig. 1, a carrier velocity of $1.4 \pm 0.2 \times 10^{4} \mathrm{~m} / \mathrm{s}$ is extracted.

found by investigating the width dependence of the characteristic quantity $I_{C} R_{N}$. A comparison of two junctions with $R_{N}=56.1$ and $51.5 \Omega$, and $W=1$ and $0.5 \mu \mathrm{m}$, (both $L=50 \mathrm{~nm}$ ) is shown in Fig. 3a, demonstrating that halving $W$ doubles $I_{C}$ despite similar values of $R_{N}$. Measurements of $R_{N}$ were carried out above the superconducting transition temperature of the leads in a four-terminal geometry, eliminating the resistance of the cryostat lines, but not the contact resistance between $\mathrm{Ti} / \mathrm{Al}$ and $\mathrm{Bi}_{2} \mathrm{Se}_{3}$, which varies from device to device without apparent correlation to geometry or effect on $I_{C} R_{N}$ product. The mismatch of the $I-V$ curves above $I_{C}$ is due to the excess current mentioned in connection with Fig. 2 (inset), which is typically of order $I_{C}{ }^{25}$ and hence larger in the $W=0.5 \mu \mathrm{m}$ device. The two curves approach each other as $V$ approaches $2 \Delta / e$ (inset Fig. 3a). The values of $I_{C} R_{N}$ for all 14 devices we measured that superconduct are shown as a function of $1 / W$ in Fig. $3 \mathrm{~b}$. The trend is clear: a larger $W$ produces a smaller $I_{C} R_{N}$. With benefit of hindsight, some previously reported experiments on TI JJs are consistent with $I_{C} R_{N}$ being related to $1 / W$. In very wide junctions no supercurrent is observed at all ${ }^{13}$. For intermediate values of $W$ similar to ours a low $I_{C} R_{N}$ was measured ${ }^{11}$. The velocity $\nu_{e x}$ of dissipative excita- 
tions in the junction can be extracted from a linear fit of the data in Fig. 3b assuming a unity proportionality constant between $I_{C} R_{N}$ and $E_{C}$ (i.e. $I_{C} R_{N}=E_{C} / \mathrm{e}$ ), giving a value $1.4 \pm 0.2 \times 10^{4} \mathrm{~m} / \mathrm{s}$. Electrical transport measurements of the Fermi velocity $\nu_{F}$ for surface electrons in $\mathrm{Bi}_{2} \mathrm{Se}_{3}$ range from $10^{5} \mathrm{~m} / \mathrm{s}{ }^{15}$ to $10^{6} \mathrm{~m} / \mathrm{s}^{11}$, bracketing the value $\nu_{e x}=4.2 \times 10^{5} \mathrm{~m} / \mathrm{s}^{26}$ extracted from ARPES, and larger than our inferred value. In fact, a lower group velocity is expected for the bound pairs of electrons and holes that shuttle Cooper pairs across the device ${ }^{27}$. Specifically for Majorana fermions - a subset of these bound pairs - the velocity $\nu_{M}$ has been predicted to be less than $\nu_{F}$ by a factor $(\Delta / \mu)^{2}$ in Ref. [4] and $(\Delta / \mu)$ in Ref. [28] (calculated for neutral modes created in graphene JJs), where $\mu$ is the chemical potential of the TI weak link relative to the Dirac point of the surface states. The typical ratio for $\Delta / \mu$ in our samples is $10^{-3}$, giving an estimate for the Majorana velocity of $0.42 \mathrm{~m} / \mathrm{s}$ for $(\Delta / \mu)^{2}$ and $4.2 \times 10^{2} \mathrm{~m} / \mathrm{s}$ for $(\Delta / \mu)$. Given that we have ignored the proportionality constants in both the relationship between $I_{C} R_{N}$ and $E_{C}$, and between $\nu_{M}$ and $(\Delta / \mu) \nu_{F}$, our results are reasonably near expectations for $\nu_{M}$ given by Ref. [28].

The last characteristic response of TI JJs considered in this Letter is the magnetic diffraction pattern. The differential response $d V / d I(B, I)$ for a $(L, W)=(55 \mathrm{~nm}$, $1.5 \mu \mathrm{m})$ device demonstrates an atypical relationship between $I_{C}$ and $B$ (Fig. 4a). Three features are of note: $B_{C}$ is 5 times smaller than expected from the known device area; the shape of $I_{C}(B)$ deviates from a typical Fraunhofer pattern; and there exist added features in $d V / d I$ at values of $I$ above $I_{C}$ whose magnitude and number depend on $B$. We consider the first two features below and address the third in $d V / d I$ in the Supp. Info. The area of the devices is calculated as $W *\left(L+2 \lambda_{L}\right)$, where $\lambda_{L}=50 \mathrm{~nm}$ is the dirty London penetration depth for aluminum (Supp. Info). The extracted $I_{C}(B)$ is shown in Fig. $4 \mathrm{~b}$ (red) and compared to the simulated Fraunhofer pattern (black) for the device area (see Supp. Info. for information on the simulation). $B_{C}$ for this device is $1.70 \mathrm{mT}$, whereas it should be $9.3 \mathrm{mT}$, based on the device area measured from a scanning electron micrograph. We have measured a smaller-than-expected value of $B_{C}$ in all our devices. The three minima in $I_{C}$ on each side of $B=0$ are unequally spaced, occurring at $B=1.70$, 6.25 , and $11.80 \mathrm{mT}$. Even if the effective area of the junction were larger for unknown reasons, fitting the central peak to a Fraunhofer pattern would produce minima at $1.7,3.4$, and $5.1 \mathrm{mT}$, different from what is observed. The graphite control device exhibits a more conventional magnetic diffraction pattern (Supp. Info).

Drastic narrowing of $I_{C}(B)$ can occur if the currentphase relation (CPR) is peaked at certain values of $\varphi$, as our phenomenological model suggests. We note that an anomalous, peaked CPR has been theoretically predicted for a different device geometry, also a result of

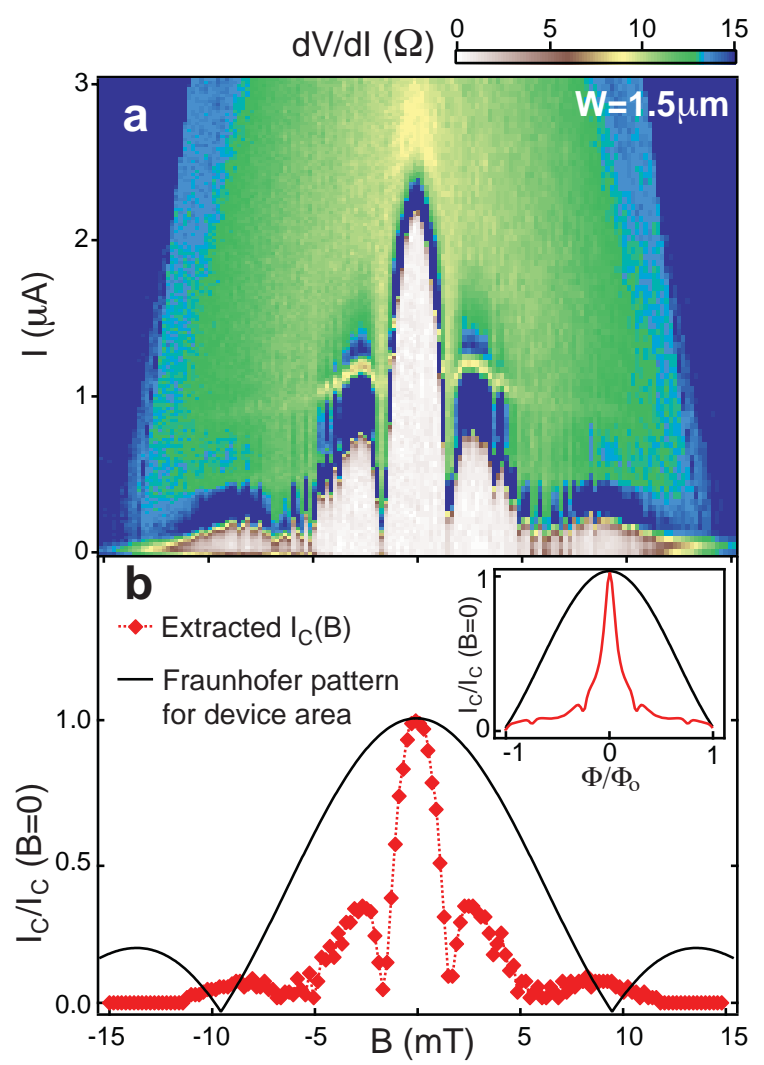

FIG. 4: Magnetic interference pattern in topological insulator Josephson junction. a, Differential resistance $d V / d I$ as a function of $B$ and $I$ showing an anomalous magnetic diffraction pattern for a $W=1.5 \mu \mathrm{m}$ junction. Two features are of note: a smaller than expected value of $B_{C}$ at $1.70 \mathrm{mT}$ and a nonuniform spacing between minima at values $B=1.70,6.50,11.80 \mathrm{mT}$. $\mathbf{b}$, (main) $I_{C}(B)$ (red) extracted from $d V / d I$ in a is compared to the expected Fraunhofer pattern for the junction (black) where a reduction of the scale of the pattern and the nonuniform spacing are evident. (inset) A comparison of the simulated Frauhofer pattern for a sinusoidal (black) and an empirically-determined, peaked (red) CPR. The aperiodic minima observed in a are captured this CPR.

the presence of Majorana fermions ${ }^{29}$. In each case, one peak in the CPR occurs for each zero-energy crossing of states in the gap. For a single peak in the CPR, only a single, $B=0$ maximum is possible for $|\Phi|<\Phi_{0}$ (Supp. Info). The existence of multiple minima in $I_{C}(B)$ for $|\Phi|<\Phi_{0}$ indicates the presence of multiple peaks in the CPR - a result of coupling to fermionic modes in the device that create additional zero-energy crossings ${ }^{30}$. For a given CPR, the resulting $I_{C}(\mathrm{~B})$ is uniquely determined; however the reverse is not true: a peak in the CPR at, for example, $\varphi=\pi / 2$ produces the same $I_{C}(\mathrm{~B})$ as a peak at $\varphi=\pi$. Through simulation, we are able to show that a smoothly-varying CPR cannot capture our results (Supp. Info) and only a CPR with peaks can create magnetic diffraction patterns like those observed in our devices. The magnetic diffraction pattern for one such peaked CPR that also includes a conventional, si- 
nusoidal contribution from the bulk is compared to the typical Fraunhofer pattern (black) in the inset of Fig. 4 b. Some, but not all, of the features observed in the experiment are captured by this CPR. Importantly it produces non-uniformly distributed minima at $\Phi_{0} / 4,3 \Phi_{0} / 4$, and $\Phi_{0}$ (see Supp. Info for details) matching the aperiodic structure of the minima seen in the experiment.

We thank Shaffique Adam, Malcolm Beasley, John Clarke, Liang Fu, Jedediah Johnson, Chris Lobb, Chetan Nayak, Xiaoliang Qi, and Victor Yakovenko for valuable discussions. The work was supported in part by the Keck Foundation. J. R. W. acknowledges support from the Karl van Bibber Postdoctoral Fellowship. A. J. B. acknowledges support from an NDSEG Fellowship. J. G. A., A. S. B. and I. R. F. are supported by the DOE, Office of Basic Energy Sciences, under contact DE-AC0276SF00515

[1] Geim, A. K. and Novoselov, K. S., The rise of graphene. Nature Mater. 6, 183-191 (2007).

[2] Yoshioka, D., The Quantum Hall Effect. Spinger-Verlag Berlin, Germany (1998).

[3] Xiaoliang, Q. and Zhang, S. C., Topological insulators and superconductors. Rev. Mod. Phys. 83, 1057-1110 (2011).

[4] Fu, L. and Kane, C. L., Superconducting proximity effect and majorana fermions at the surface of a topological insulator. Phys. Rev. Lett. 100, 096407 (2008).

[5] Teo, J. C. Y. and Kane, C. L., Majorana fermions and non-abelian statistics in three dimensions, Phys. Rev. Lett. 101, 046401 (2010).

[6] Kitaev, A., Fault-tolerant quantum computation by anyons. arXiv:quant-ph/9707021 (1997).

[7] Wilczek, F., Majorana returns. Nature Phys. 5, 614-618 (2009).

[8] Rajasekaran, G., Are neutrinos majorana particles? arXiv:0803.4387 (2008).

[9] Sasaki, S., et al., Topological superconductivity in $\mathrm{Cu}_{x} \mathrm{Bi}_{2} \mathrm{Se}_{3}$. Phys. Rev. Lett. 107, 217001 (2011).

[10] Koren, G., Kirzhner, T., Lahoud, E., Chashka, K. B. and Kanigel, A., Proximity-induced superconductivity in topological $\mathrm{Bi}_{2} \mathrm{Te}_{2} \mathrm{Se}$ and $\mathrm{Bi}_{2} \mathrm{Se}_{3}$ films: Robust zeroenergy bound state possible due to Majorana fermions. Phys. Rev. B. 84224521 (2011).

[11] Sacépé, B., et al., Gate-tuned normal and superconducting transport at the surface of a topological insulator. Nature Comm. 2 575, (2011).

[12] Zhang, D., et al., Superconducting proximity effect and possible evidence for Pearl vortices in a candidate topological insulator. Phys. Rev. B 84, 165120 (2011).

[13] Wang, J., et al., Interplay between topological insulators and superconductors. Phys. Rev. B 85, 045415 (2012).

[14] Qu, F., et al., Strong superconducting proximity effect in $\mathrm{Pb}_{-} \mathrm{Bi}_{2} \mathrm{Se}_{3}$ Hybrid Structures. arXiv:112.1683.

[15] Veldhorst, M., et al., Josephson supercurrent through a topological insulator surface state. arXiv:112.3527.

[16] Tinkham, M., Introduction to Superconductivity. Dover Publications, Mineola, New York (1996).
[17] Fu, L. and Kane, C. L., Josephson current and noise at a superconductor/quantum-spin-Hallinsulator/superconductor junction. Phys. Rev. B 79, 161408 (2009)

[18] Fazio, R., Hekking, F. W. J., and Odintsov, A. A., Josephson current through a Luttinger liquid. Phys. Rev. Lett. 74, 1843-1846 (1995).

[19] Maslov, D. L., Stone, M., Goldbart, P. M., Loss, D., Josephson current and proximity effect in Luttinger liquids. Phys. Rev. B. 53, 1548-1557 (1996).

[20] Schon, G. and Zaikin, A. D., Quantum coherent effects, phase transitions, and the dissipative dynamics of ultra small tunnel junctions. Phys. Rep. 198, 237-412 (1990).

[21] Kane, C. L. and Fisher, M. P. A. Fisher, Transport in a one-channel Luttinger liquid. Phys. Rev. Lett. 68, 12201223 (1992).

[22] Analytis, J. G., et al., Two-dimensional surface state in the quantum limit of a topological insulator. Nature Phys. 6, 960-964 (2010).

[23] Peng, H., et al., Aharonov-Bohm interference in topological insulator nanoribbons. Nature Mater. 9, 225-229 (2010).

[24] Flensberg, K., Bindslev Hansen, J., and Octavio, M., Subharmonic energy-gap structure in a superconductor weak link. Phys. Rev. B 38, 8707-8711 (1988).

[25] Likharev, K. K., Superconducting weak links. Rev. Mod. Phys. 51, 101-159 (1979).

[26] Analytis, J. G., et al. Bulk fermi surface coexistence with Dirac surface state in $\mathrm{Bi}_{2} \mathrm{Se}_{3}$ : A comparison of photoemission and Shubnikov de Haas measurements. Phys. Rev. B 81, 205407 (2010).

[27] Shytov, A. V., Lee, P. A., and Levitov, L. S., Localization of quasiparticles in an NS structure. Phys. Usp. 41, 207210 (1998).

[28] Titov, M., Ossipov, A., and Beenakker, C. W. J., Excitation gap of a graphene channel with superconducting boundaries. Phys. Rev. B 75, 045417 (2007).

[29] Ioselevich, P. A. and Feigel'man, M. V. Anomalous Josephson Current via Majorana Bound States in Topological Insulators. Phys. Rev. Lett. 106, 077003 (2011).

[30] Law, K. T. and Lee, P. A., Robustness of Majorana fermion induced fractional Josephson effect in multichannel superconducting wires. Phys. Rev. B 84, 081304 (2011). 


\section{Supplementary Information for Signatures of Majorana Fermions in Hybrid \\ Superconductor-Topological Insulator Devices}

\section{Materials and methods}

Single crystals, grown by the binary melt method, of $\left(\mathrm{Bi}_{1-x} \mathrm{Sb}_{x}\right)_{2} \mathrm{Se}_{3}$ were synthesized by slow cooling a binary melt of $\mathrm{Bi}$ (99.9999\%), $\mathrm{Sb}(99.999 \%)$ and Se $(99.9999 \%)$ starting materials, mixed in the ratio 0.33:0.05:0.62. The actual amount of $\mathrm{Sb}$ in the single crystals is approximately 0.01 , as measured in microprobe analysis. Bulk single crystals with dimensions $1 \times 1 \times 0.1 \mathrm{~mm}^{3}$ showed a non-metallic resistivity at low temperatures with a bulk carrier density of $7 \times 10^{16} \mathrm{~cm}^{-3}$ as deduced by Hall and Shubnikov-de Haas analysis.

$\mathrm{Bi}_{2} \mathrm{Se}_{3}$ nanowires, synthesized via the vapor-liquidsolid (VLS) method, were grown in a 12-inch horizontal tube furnace with a quartz tube. $\mathrm{Bi}_{2} \mathrm{Se}_{3}$ source powder (99.999\%, from Alfa Aesar) was placed in the center of the furnace $\left(540^{\circ} \mathrm{C}\right)$. The growth substrate, a $\mathrm{Si}$ wafer with a thermally-evaporated, $10 \mathrm{~nm} \mathrm{Au} \mathrm{film,} \mathrm{was} \mathrm{placed}$ at a downstream, lower temperature zone $\left(350^{\circ} \mathrm{C}\right)$. High purity Ar gas delivers vapor from the source materials to a growth substrate at $130 \mathrm{sccm}$ for 2 hours with 1 Torr pressure.

Samples of 50-100 nm thickness were prepared by mechanical exfoliation of the above material, using a method similar to exfoliation of graphene ${ }^{S 1}$. Prior to sample exfoliation, $\mathrm{SiO}_{2}(300 \mathrm{~nm}) / \mathrm{Si}$ wafers were cleaned in acetone and isopropanol. HMDS was deposited on the $\mathrm{SiO}_{2}$ surface in efforts to reduce the amount of $\mathrm{H}_{2} \mathrm{O}$ contamination. Samples were exfoliated using Nitto-Denko tape and immediately after exfoliation PMMA 950 A4 (Microchem Crop.) was spun on the chip for 60 secs at $4000 \mathrm{rpm}$, followed by a baked for $30 \mathrm{mins}$ at $180^{\circ} \mathrm{C}$. After electron beam exposure of the resist and development in MIBK/IPA 1:3, a 3 min UV-ozone was used to remove residual PMMA in the exposure contact areas. Ion milling of the exposed contact areas was performed to obtain low contact resistance to the $\mathrm{Bi}_{2} \mathrm{Se}_{3}$ flakes. The recipe of the ion mill is a $300 \mathrm{~V}$ beam voltage, $30 \mathrm{~mA}$ beam current producing an ion current density of $0.1 \mathrm{~mA} / \mathrm{cm}^{2}, 60 \mathrm{~V}$ acceleration, $12 \mathrm{sccm}$ argon flow, and a 10 secs exposure. Immediately following ion milling, the samples were loaded into e-beam evaporator with oil-free pumps and $\mathrm{Ti} / \mathrm{Al}(3 \mathrm{~nm}$ and either $60 \mathrm{~nm}$ or $100 \mathrm{~nm})$ were evaporated at a rate of $1 \AA / \mathrm{s}$ onto the sample at a pressure of $\sim 5 \times 10^{-8}$ Torr at the start of each evaporation.

Samples were measured in a ${ }^{3} \mathrm{He} /{ }^{4} \mathrm{He}$ dilution refrigerator with a base temperature of $12 \mathrm{mK}$. To reduce the effects of thermal radiation, three stages of filtering were employed: room-temperature LC $\pi$-filters, a 3-pole RC filter anchored to the mixing chamber designed to filter in the frequency range of $1 \mathrm{MHz}$ to $5 \mathrm{GHz}^{S 2}$, and $10 \mathrm{k} \Omega$ "on-chip" resistors used in conjunction with the inline capacitance of the twisted-pair wiring to produce a lowpass, RC-filter of cutoff frequency $\sim 50 \mathrm{KHz}$. All experiments were performed in an electrically- and acousticallyshielded room. Device DC resistance and differential resistance were measured using a standard DC and lock-in technique. The applied AC current was kept low enough such that the AC voltage across the device never exceeded $500 \mathrm{nV}$, below the base temperature of the fridge $\mathrm{k}_{B} \mathrm{~T} \sim 1 \mu \mathrm{eV}$. To obtain accurate magnetic field values, a low-noise Yokogawa 7651 was used to supply the current to the superconducting magnet, producing a $<0.3 \mu \mathrm{T}$ noise level in the magnet.

The transport properties of the exfoliated binary melt samples were characterized by making a device with contacts in a Hall-bar geometry and thickness of $100 \mathrm{~nm}$. The extracted density, mobility and diffusion constant $D$ of these samples are $7.8 \times 10^{17} \mathrm{~cm}^{-3}, 1950 \mathrm{~cm}^{2} / \mathrm{Vs}$, $0.02 \mathrm{~m}^{2} / \mathrm{sec}$, respectively. We note that these values reflect merged contributions from both the surface and bulk, where it is expected that the surface electrons have a much higher mobility ${ }^{S 3}$. Hence, the values for mobility and $D$ are a lower bound for the values of the surface electrons.

To extract the superconducting and normal state properties of the leads, four samples were prepared with the same recipe as the leads of the JJ devices: $\mathrm{Ti} / \mathrm{Al}$ of thickness $3 \mathrm{~nm} /(60,100 \mathrm{~nm})$, both of $(L, W)=(10,1) \mu \mathrm{m}$. Each thickness of $\mathrm{Al}$ was placed either directly on the $\mathrm{SiO}_{2}$ substrate or on top of a $\mathrm{Bi}_{2} \mathrm{Se}_{3}$ flake. Characterization of these test samples was performed via a fourterminal resistance measurement at room temperature $(290 \mathrm{~K}), 4 \mathrm{~K}$ and base temperature of the dilution refrigerator $(12 \mathrm{mK})$. Little variation was found in the superconducting properties of the $\mathrm{Al}$ patterned on the $\mathrm{SiO}_{2}$ substrate and on $\mathrm{Bi}_{2} \mathrm{Se}_{3}$. The resistivity of the samples at room temperature and $4 \mathrm{~K}$ were $3.0 \times 10^{-8} \Omega-\mathrm{m}$ and $0.3 \times 10^{-8} \Omega$-m, respectively. The critical currents and magnetic fields for the $100 \mathrm{~nm}(60 \mathrm{~nm})$-thick Al-films were $488 \mu \mathrm{A}(208 \mu \mathrm{A})$ and $11(13) \mathrm{mT}$ respectively.

\section{Characteristic lengths and regimes for JJs}

There are many different regimes in JJs that produce different behaviors. The regime applicable to the device is determined by the length of the junction $L$ relative to five intrinsic length scales: coherence length of the $\mathrm{Al}$ leads $\xi_{A l}=1.6 \mu \mathrm{m}$; the superconducting coherence length of the metallic $\left(\mathrm{Bi}_{2} \mathrm{Se}_{3}\right)$ weak link $\xi_{N}$, and three properties of the $\left(\mathrm{Bi}_{2} \mathrm{Se}_{3}\right)$ weak link unrelated to the superconductor: the thermal length $\ell_{T}$, the mean-free path $\ell_{e}$ and the phase-coherence length $\ell_{\varphi} . \quad \xi_{N}$ can be calculated from the diffusion constant by $\sqrt{\hbar D / \Delta}$, where $\Delta$ is the superconducting gap of $\mathrm{Al}$, which we calculate from the BCS equation $\Delta=1.76 k_{B} T_{C}=151 \mu \mathrm{eV}$, producing a value for $\xi_{N}$ of $\sim 280 \mathrm{~nm}$. $\ell_{T}=\sqrt{\hbar D / 2 \pi k_{B} T}$, 
which at $12 \mathrm{mK}$ is $1.3 \mu \mathrm{m}$. $\ell_{e}$ can be extract from $\mathrm{D}$ as $\mathrm{D}=1 / 2 \nu_{F} \ell_{e}$, giving $\ell_{e}=80 \mathrm{~nm}$, where we have used the value of $\nu_{F}=4.2 \times 10^{5} \mathrm{~m} / \mathrm{s}$ from ARPES measurements ${ }^{S 4}$. $l_{\varphi}$ was estimated from the half-width at half-max of the weak antilocalization correction to the longitudinal resistance (measured in the Hall bar) to be $650 \mathrm{~nm}$. We note that $\xi_{N}, \ell_{T}, \ell_{e}$ and perhaps $\ell_{\varphi}$ all represent lower bounds since we have used the average value of $D$ from the surface and the bulk. The $D$ for the surface electrons is expected to be higher. Further, $W$ for the device should be compared to the Josephson penetration depth $\lambda_{J}=\sqrt{\Phi_{\mathrm{o}} /\left(2 \pi \mu_{\mathrm{o}} j_{C}\left(2 \lambda_{L}+L\right)\right)}$, where $\lambda_{L}$ is the London penetration depth $\sim 50 \mathrm{~nm}^{S 5}$. We place a lower bound on $\lambda_{J}$ by using the junction parameters that would make it the smallest, i.e. using the largest measured value of $j_{C}$ and the longest value of $L$ : producing a value for $\lambda_{J}^{\min }$ of $10 \mu \mathrm{m}$. This puts our junctions in the regime $\mathrm{L} \sim \ell_{e}<\xi_{N}, \xi_{A l}, \ell_{\varphi}, L_{T}$ and $W<\lambda_{J}$, i.e. the short junction, quasi-ballistic regime. In this regime, $I_{C} R_{N}$ is expected to be described by either the $\mathrm{KO}-1$ or $\mathrm{KO}-2$ theory $^{S 6}$ and independent of $W$, neither of which expectation matches our data.

\section{Supercurrent carried by the bulk}

The typical thickness $t$ for our devices is $75 \mathrm{~nm}$. The calculated $\xi_{N}$ is larger than this value, which means that both the surface and entire bulk should experience the proximity effect. We expect, however, that the supercurrent carried by the bulk to be smaller than that of the surface for two reasons. First, the Cooper pairs have to travel a longer distance from left to right lead through the bulk than through the surface. We can estimate this additional length by assuming the Cooper pairs have to go on average a distance $t / 2$ from the left lead to the bulk, then another $t / 2$ going from the bulk to the right lead. This makes the effective length of the device for the bulk states to be $L_{b u l k}=L+t$. The second is the lower mobility of the bulk, producing a smaller value of $D$. For the device in Fig. 4 of the main manuscript $L=55 \mathrm{~nm}$ and $\mathrm{t}=95 \mathrm{~nm}$, making $L_{b u l k}=150 \mathrm{~nm}$. To calculate the reduction of the critical current, we assume $D$ for the bulk states is 10 times smaller than the calculated value (a factor 12 has been experimentally determined ${ }^{S 7}$ for the reduction of $D$ for the bulk), giving a $\lambda_{N}^{b u l k} \sim 65 \mathrm{~nm}$. This estimate yields a bulk critical current of order five times smaller than that of the surface state ${ }^{S 6}$.

\section{Mechanisms for the reduction of $I_{C} R_{N}$}

We next consider the other possible mechanisms for the reduction of $I_{C} R_{N}$. A typical reduction comes from thermal noise, where the effect on $I_{C} R_{N}$ depends on the type of junction (overdamped or underdamped). The
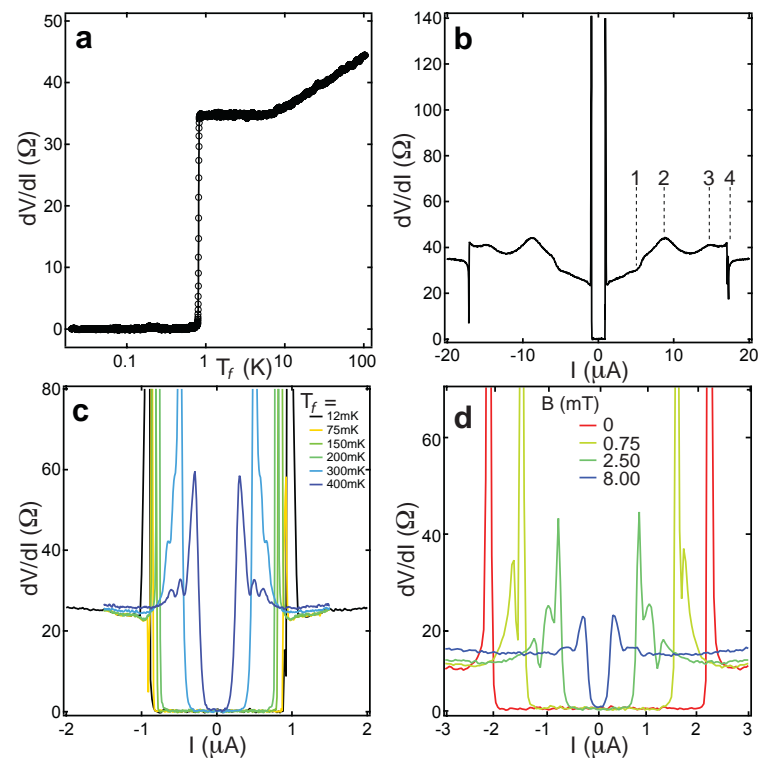

FIG. 5: Additional features in $d V / d I$ as function of temperature, $\boldsymbol{I}_{D C}$, and $\boldsymbol{B}$. a, Differential resistance $d V / d I$ as a function of temperatures $T_{f}$ as $T_{f}$ is lower through $T_{C}$ of the leads. For this device, a sharp transition to the superconducting state is observed at $T_{f}=850 \mathrm{mK}$. b, Additional features in $d V / d I$ as a function of $I$ are observed above $I_{C} . \mathbf{c}, d V / d I$ for values of $T_{f}=12,75,150,300$, $400 \mathrm{mK}$. Shoulder dips are observed in $d V / d I$ for the two temperatures 300 and $400 \mathrm{mK}$. d, $d V / d I$ extracted for $\mathrm{B}=0,0.75,2.50$, $8.00 \mathrm{mT}$ from Fig. 4a of the main text. Shoulder dips are also apparent when the magnetic field is increased to $0.75 \mathrm{mT}$.

type of junction is determined the quality factor $Q=$ $\sqrt{2 e I_{C} C / \hbar} R_{N}$, where $C$ is the capacitance of the JJ. We estimate $C$ to be $0.5 \mathrm{pF}$ using a parallel plate capacitor model between the entire area of the leads of the device and the degenerately-doped $\mathrm{Si}$, yielding $Q=1.2$. This is neither overdamped or underdamped, but at an intermediate value. Numerical evaluation of the $I-V$ characteristics in this regime shows results similar to those of an overdamped junction ${ }^{58}$. The effect of thermal radiation on overdamped junctions has been calculated ${ }^{S 8}$, and shown to cause a smearing of the transition between the superconducting and normal state. Since the transitions we measure are still very sharp, we can rule out thermal fluctuations being a source of the reduction of $I_{C} R_{N}$. We can also calculate what the effect of thermal fluctuations would be had the junctions been in the underdamped regime ${ }^{S 5}$ : the effective thermal radiation temperature would have to be $3.4 \mathrm{~K}$ to reduce $I_{C} R_{N}$ by the factors we observe. This high temperature is unphysical in our well-filtered setup.

\section{Additional features in the Differential Response $\mathrm{dV} / \mathrm{dI}$ as a function of $\mathrm{T}$ and $\mathrm{B}$}

The differential response $d V / d I$ for a $(L, W)=(50 \mathrm{~nm}$, $0.9 \mu \mathrm{m})$ device as a function of fridge temperature $T_{f}$ 
is shown in Fig. S1a, where the device exhibits a sharp transition to $d V / d I=0$ below $T_{f}=850 \mathrm{mK}$ of the leads. Above $\mathrm{T}_{C}$, the devices shows a metallic temperature dependence of resistance. The differential response $d V / d I(I)$ of this junction, typical for all devices measured, at $T_{f}=12 \mathrm{mK}$ is shown in Fig. S1b. Even aside from the transition from a superconducting state to a normal state, which produce large peaks at $I=I_{C}$, several features are evident and are indicated by labels 1-4. The first occurs in all devices as either a peak or a sharp rise in $d V / d I$ (like the one shown in Fig. $\mathrm{S} 1 b)$, at $V=200 \mu \mathrm{V}$. The second, broad peak appears at $V=300 \mu \mathrm{V}$, near $2 \Delta / \mathrm{e}$ of $\mathrm{Al}$, where a peak in resistance is expected due to the quasiparticle contribution to the resistance from the leads ${ }^{S 9}$. The third and fourth peaks are both features occurring above $2 \Delta$ of the leads, occurring at $V=500 \mu \mathrm{V}$ and $V=655 \mu \mathrm{V}$, respectively. Peaks above $2 \Delta$ have also been seen previously in TI $\mathrm{JJS}^{S 10}$.

The effect of $T_{f}$ on $d V / d I$ is shown in Fig. S1c. A typical trend is observed, where increasing $T_{f}$ reduces $I_{C}$. Additional "shoulder" dips appearing in $d V / d I$ are evident in the $T_{f}=300 \mathrm{mK}$ and $400 \mathrm{mK}$ traces. On the $400 \mathrm{mK}$ trace, two dips are seen, at $I=500 \mathrm{nA}$ and $620 \mathrm{nA}$ respectively.

Additional shoulder features are also seen in $d V / d I$ above $I_{C}$ when a magnetic field $B$ is applied (Fig. S1d). These are revealed in cuts of constant $B$ in $d V d I(B, I)$ for the device shown in Fig. 4 of the main text. At $B=0$, there are two peaks in $d V / d I$ at $I= \pm I_{C}$, and no other apparent features. At $B=0.75 \mathrm{mT}$, dips are evident at $I= \pm 1.6 \mu \mathrm{A}, V=12.5 \mu \mathrm{V}$ above the resistive transition. The single dip occurs for $0.6 \leq B \leq 1 \mathrm{mT}$. Two dips appear beginning at $B=1 \mathrm{mT}$ and persist throughout the entire second and third lobes, although to a much weaker degree in the third lobe. The double-dip is evident in the cut at $B=2.5 \mathrm{mT}$, occurring at $I \pm=0.9$ and $1.2 \mu \mathrm{A}$ and a value of $V=7.4$ and $12.1 \mu \mathrm{V}$. The dips at $B=8 \mathrm{mT}$ have all but disappeared, remaining weakly at $I \pm=0.5$ and $0.9 \mu \mathrm{A}$ and $V=6.3$ and $12.1 \mu \mathrm{V}$. Currently, we have no explanation for these features which appear in all devices measured.

\section{Andreev Bound States and the Current-Phase Relation}

In this section we consider qualitatively the Andreev bound states (ABS) and the resulting current-phase relation (CPR). States in a JJ within the superconducting gap are associated with the coupled electron-hole pairs that are responsible for transferring Cooper pairs from one lead to the other ${ }^{S 11}$. These are known as ABS. To describe the CPR in our junction, we consider several different energy spectrum of ABS. The energy levels of conventional ABS are $E(\varphi) \propto \pm \Delta \sqrt{1-T \sin ^{2}(\varphi / 2)} S 11$, where $T$ is the transmission probability of Cooper pairs
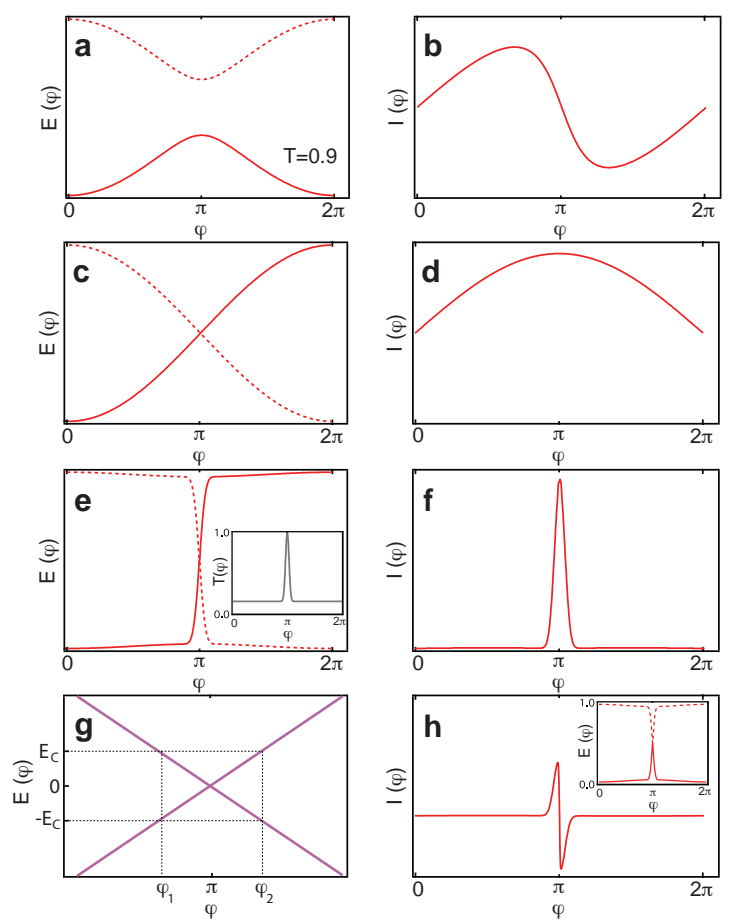

FIG. 6: Determining the Current-Phase Relation from the Andreev Bound State Energy Spectrum. The CPR can be determined from the Andreev bound state (ABS) energy spectrum by taking the derivative of $E$ with respect to $\varphi$. Qualitatively shown is the ABS spectrum (a) and the corresponding CPR (b) for conventional, ballistic JJs with a transmission probability $T=0.9$. The same plots $(\mathbf{c}, \mathbf{d})$ are shown for a $p+i p$ and TI (without the lateral confinement of our model) JJs. ABS spectrum and CPR $(\mathbf{e}, \mathbf{f})$ for the phenomenological model of the main text. The inset of e shows the dependence of $T$ on $\varphi$ in our model. The CPR of (f) is peaked at the values of $\varphi$ where the zero-energy crossings occur. $\mathbf{g}$, The width of $T$ in the inset of $\mathbf{e}$ and the corresponding CPR are set by $E_{C}$, the lateral confinement energy in the phenomenological model. $\mathbf{h}$, (inset) ABS spectrum similar to $\mathbf{S 2 e}$ except with a anticrossing in the spectrum at $\varphi=\pi$. (main) CPR resulting from the ABS of the inset.

from left to right lead. From the energy spectrum of the ABS, the current phase relation (CPR) can be calculated as $I(\varphi) \propto \partial E / \partial \varphi^{S 10}$. The energy spectrum for the ABS in a conventional JJ with $T=0.9$ is shown in Fig. S2a for the occupied (solid) and unoccupied (dashed) ABS states. The corresponding CPR, calculated by differentiating the $E(\varphi)$ plot, is shown in Fig. S2b, where a saw-tooth behavior is seen, occurring with a period of $2 \pi$. Note that for low values of $T$, this CPR becomes the conventional $\sin (\varphi)^{S 11}$. For $p+i p$ and TI JJs, the energy of the ABS becomes $E(\varphi) \propto \pm \Delta \cos (\varphi / 2)$, producing a zero-energy crossing at $\varphi=\pi^{S 12}$. There is a correspondence between the $p+i p$ and conventional energy spectrum of ABS for $T=1$. The corresponding CPR is $I(\varphi) \propto \sin (\varphi / 2)$, resulting in an anomalous $4 \pi$ periodic CPR, predicted for $p+i p \mathrm{JJs}^{S 12}$ and $\mathrm{TI}^{S 13} \mathrm{JJs}$. The energy spectrum of the ABS and the CPR in this case are shown in Fig. S2c,d. 
In our phenomenological model, the transmission coefficient $T$ depends on $\varphi$, where the 1D charge modes at $\varphi \neq \pi$ have a low value for $T-$ a result of the interactions in $1 \mathrm{D}^{S 14}$ - and the neutral modes have a much higher value for $T$. This $T(\varphi)$ produces a CPR that is peaked at values of $\varphi$ where the zero-energy modes occur. Fig. S2e shows an ABS spectrum for a $T(\varphi)$ (inset of Fig. S2e), corresponding to $T(\varphi)=1$ for $\varphi=\pi$ and $T(\varphi)=1 / 10$ for $\varphi \neq \pi$. The exact value for $T$ away from $\varphi=\pi$ can be changed without much change in the results . To connect the high- and low- $T$ regions, we assume that the width of the peak in $T(\varphi)$ near $\pi$ is set by the energy scale $E_{C}$, the energy associated with momentum quantization in the phenomenological model. This scenario is shown in Fig. S2g, where the linearized $E(\varphi)$ is calculated from Eq. 4 of Ref. [S15]. Using a value of $E_{C}=e I_{C} R_{N}=31 \mu \mathrm{eV}$ for the device in Fig. 4, values of $167^{\circ}$ and $193^{\circ}$ for $\varphi_{1}$ and $\varphi_{2}$ are obtained. $T$ then is modeled as a gaussian with a half max at the values $\varphi_{1}$ and $\varphi_{2}$. The calculated ABS spectrum for this transmission coefficient is shown in Fig. S2e, where a transition through $E=0$ occurs on the scale of $\varphi_{1}$ and $\varphi_{2}$ around $\varphi=\pi$. The corresponding $I(\varphi)$ is shown in Fig. S2f, where is it seen that the $\varphi$-dependent $T$ produces a anomalous CPR that is peaked around the values of $\varphi$ where the zero-energy modes occur. This CPR is also $4 \pi$ periodic. A similar CPR was obtained in Ref. S16, where a peak in the CPR was also found to be correlated with the zero-energy crossing in the ABS. In Fig. S2c and S2e, it was assumed that sermonic parity was conserved, which results in a protected crossing of the two states (solid and dashed lines) at $\varphi=\pi^{S 17}$. If the parity of the junction is not preserved, an anti-crosssing of these two states occurs (inset of Fig. S2h), producing a CPR shown in Fig. S2h.

\section{Simulation of Josephson Effect in the Presence of Magnetic Flux}

The critical current through the devices as a function of applied field was calculated in a manner closely following that of Tinkham ${ }^{S 5}$, but with the extended junction model modified to allow non-standard current-phase relationships and spatial inhomogeneities critical current density. With the flake surface parallel to the $x-y$ plane, applied field $B$ along $z$, and current along $x$, the phase difference between the leads as a function of the position $\mathrm{y}$ along the junction is given by

$$
\varphi(y)=\varphi_{0}+\frac{2 \pi}{\Phi_{0}} \int_{0}^{y} d y^{\prime} \int_{0}^{L+2 \lambda_{L}} d x B_{z}\left(x, y^{\prime}\right)
$$

where $\varphi_{0}$ is the phase difference at $\mathrm{y}=0, \Phi_{0}$ is the magnetic field flux quantum, $L$ is the length of the junction, and $\lambda_{L}$ is the London penetration depth. This follows
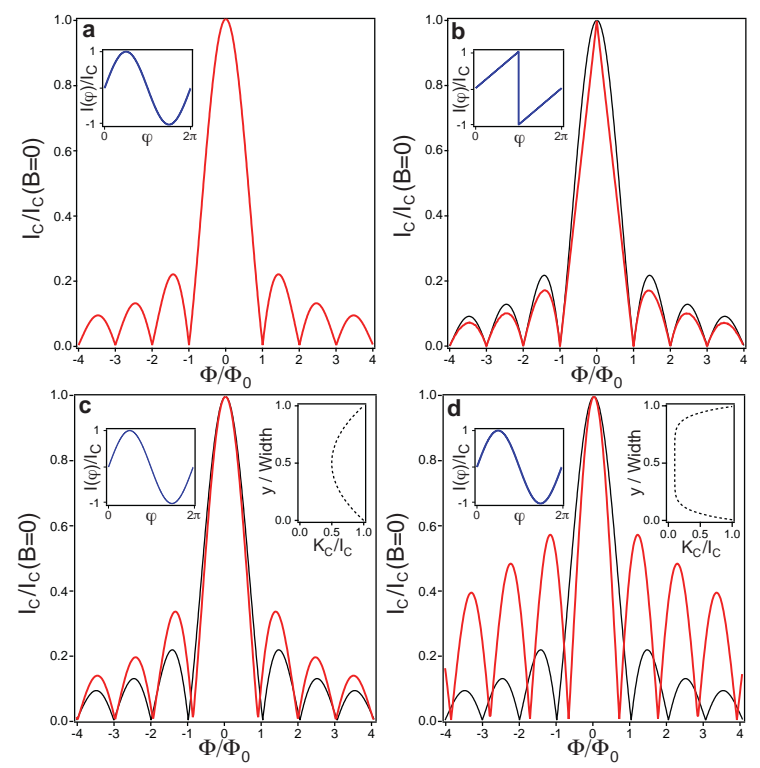

FIG. 7: Magnetic diffraction patterns for conventional current-phase relations. Magnetic diffraction patterns (red) for a, sinusoidal $I(\varphi)$, b saw-toothed sinusoidal $I(\varphi), \mathbf{c}, \mathbf{d}$, sinusoidal $I(\varphi)$ with two different nonuniform critical current line densities $K_{c}$. In b-d, the magnetic diffraction pattern for the sinusoidal $I(\varphi)$ (black) from a is plotted for comparison. In each figure, the current-phase relations used to produced the magnetic diffraction pattern is shown in the inset.

from integrating the vector potential $\mathbf{A}$ around a rectangular contour that includes a path at $\mathrm{y}^{\prime}=0$ and $\mathrm{y}^{\prime}=\mathrm{y}$ and taking advantage of the fact that $\mathbf{A}=\left(\Phi_{0} / 2 \pi\right) \nabla \gamma$ inside the superconducting leads, where $\gamma$ is the non-gaugeinvariant phase [defined so that $\varphi=\Delta \gamma-\left(2 \pi / \Phi_{0}\right) \int \mathbf{A}$. $d \mathbf{s}$. With normalized current-phase relationship (CPR) $i(\varphi)=I(\varphi) / I_{C}$ and critical current line density $K_{c}(y)$, we obtain critical current

$$
I_{C}=\max _{\varphi_{0}} \int_{0}^{W} d y K_{c}(y) i(\varphi(y)) .
$$

Note that this analysis is equally valid for $2 \pi$ - and $4 \pi$ periodic phase relations, so long as for the latter case we allow $\varphi(y)$ to range up to $4 \pi$ and define $i(\varphi)$ over the full range. For a typical $2 \pi$-periodic current-phase relationship $I(\varphi)=I_{C} \sin (\varphi)$ and uniform current density and field, the critical current drops to zero when an integer number of flux quanta are threaded through the junction area, corresponding to an integer number of cycles of Josephson current. A simulation of magnetic diffraction pattern (MDP) for $i(\varphi)=\sin (\varphi)$ shows the typical Fraunhofer pattern ${ }^{S 5}$ (Fig. S3a). Deviations from this typical pattern can occur because of non-sinusoidal CPR or from nonuniform critical current distribution along the junction. For a ballistic JJ, the CPR becomes more of a saw-toothed shape, for which the corresponding MDP is shown in Fig. S3b. There is a small change in the shape of the MDP, but the minima still occur at integer multiples of $\Phi_{0}$. Aside from a changing CPR, deviations from 

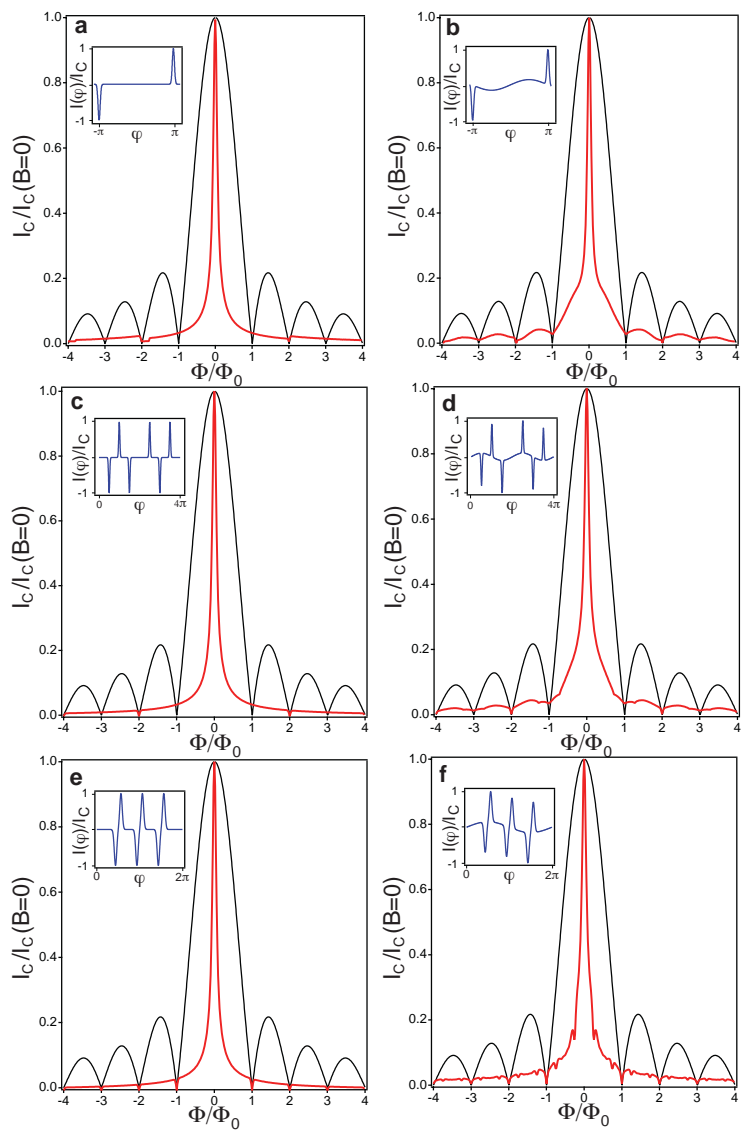

FIG. 8: Magnetic diffraction patterns for anomalous current-phase relations. Magnetic diffraction patterns (red) for a two gaussian peaks in $I(\varphi)$ at $\varphi= \pm \pi$. b, the same as a but with an added conventional sinusoidal $I(\varphi)$ of $1 / 5$ the amplitude of the anomalous peaks, $\mathbf{c}$, the same $\mathbf{a}$, with additional peaks at $\varphi=\pi / 2,3 \pi / 2$ and $5 \pi / 2,7 \pi / 2$, resulting from additional zero-energy crossings. $\mathbf{d}$, the same as $\mathbf{c}$ but with a conventional sinusoidal $I(\varphi)$ of $1 / 5$ the amplitude of the anomalous peaks. In each figure, the current-phase relations used to produced the magnetic diffraction pattern are shown in the inset and each diffraction pattern is compared to the conventional sinusoidal case (black). e, A peaked CPR for an ABS spectrum in Fig. S2h, with additional zero-energy crossings at $\varphi=\pi / 2, \pi$ and $3 \pi / 2$. f, same as except with the sinusoidal bulk contribution added.

the Fraunhofer pattern for short junctions can occur for a non-uniform current distribution. Reductions of the the location of the first minimum as large as a factor of 2 can occur in the extreme limit when all of the current is concentrated near the end of the junction ${ }^{S 18}$. The MDP in this extreme case is shown for two different current distributions in Fig. S3c, d. None of these deviations produce a MDP similar to Fig. 4 of the main text.

Using this simulation, we empirically determine a CPR to match the MDP we observe in our devices. Not any CPR is possible: the CPR must be antisymmetric $I(\varphi)=-I(-\varphi)$, it must come from the derivative of the energy of the junction and must average to zero over a period such that the energy of the junction $E(\varphi) \propto \int d \varphi I(\varphi)$ does not continue to grow as a func- tion of $\varphi^{S 19}$. In addition, the CPR must contain a term for the contribution of the bulk, which was estimated in our devices to be smaller than the surface, but not zero. In the following, we assume the bulk has a CPR $I(\varphi) \propto \sin (\varphi)$. To begin, we assume the simplest CPR that is peaked at values of $\pi$ and $3 \pi$, with alternating signs but equal amplitude to satisfy the conditions on the CPR. This result is shown in Fig. S4a, where we see that the inclusion of peaks at $\varphi=\pi, 3 \pi$ produces a narrowed MDP. It does not, however, reproduce the additional minima seen in Fig. 4. Note that this CPR is $4 \pi$-periodic, as expected for TI JJs. The first correction to the simple, peaked CPR above comes from the addition of the bulk term, which we include as a simple sinusoidal dependence on $\varphi, \sin (\varphi)$, with $1 / 5$ the amplitude (as estimated above) of the peaks in the CPR and is $2 \pi$-periodic. Compared to Fig. S4a, additional features in the MDP, are thereby introduced at $n \Phi_{0}$, where $n$ is an integer. To get closer to the observed MDP, we add more zero-energy crossings, predicted in Ref. [S17]. We add two additional zero-energy modes or either side of $\varphi=\pi$ and $3 \pi$, at $\varphi=\pi / 2,3 \pi / 2$ and $5 \pi / 2,7 \pi / 2$, and the corresponding MDP is shown in Fig. S4c,d. The MDP is calculated without (S4c) and with (S4d) the $\sin (\varphi)$ contribution from the bulk. It is seen in Fig. S4d, a small notch in the MDP appears below the first expected minima, occurring at a value of $3 \Phi_{0}$. A CPR similar to that used in Fig. S3c,d was calculated as an anomalous contribution to the CPR from Majorana fermions ${ }^{S 16}$, a result of difference in Josephson current from the even and odd Majorana occupancies in the JJ. The last CPR considered are of the form from Fig. S2h and are shown in Fig. $\mathrm{S} 4 \mathrm{e}, \mathrm{f}$, where we again have used the multiple zero-energy crossings of Ref. [S17]. The bulk importance of the inclusion of a bulk term is evident here, where without the sinusoidal addition no sub- $\Phi_{0}$ dips occur (Fig. S4e). The MDP of Fig. S4f produces minima at values $\Phi_{0} / 4,3 \Phi_{0} / 4$ and $\Phi_{0}$, near the experimentally observed minima. We note that we cannot determine a unique CPR from the diffraction pattern but can only supply evidence for a peaked CPR. In our simulations, we were unable to obtain sub- $\Phi_{0}$ dips for any uniform (i.e. non-peaked) CPR, but were only able to obtain MDPs that look similar to our experimental results using peaked CPRs.

MDPs for a varying bulk contribution are shown in Fig. S5. Fig. S5a is a 2D plot of the MDP for the CPR of Fig. S4e as a function of $\Phi$ and the amplitude of the sinusoidal contribution (Sine Amp). When Sine Amp=0 (all surface), the resulting MDP is the same as Fig. S4e, while for Sine Amp=1 (all bulk) the MDP is same as Fig. S3a. Fig. S5a shows that sub- $\Phi_{0}$ minima are obtained for values of Sine Amp less than $\sim 0.4$. For Sine $A m p=0.5$ (red), the MDP obtained is shown in Fig. S5b, where it is seen that a narrowing of the central feature occurs when compared to a Fraunhofer pattern (black), but the minima still occur at integer values of $\Phi_{0}$. A MDP similar 

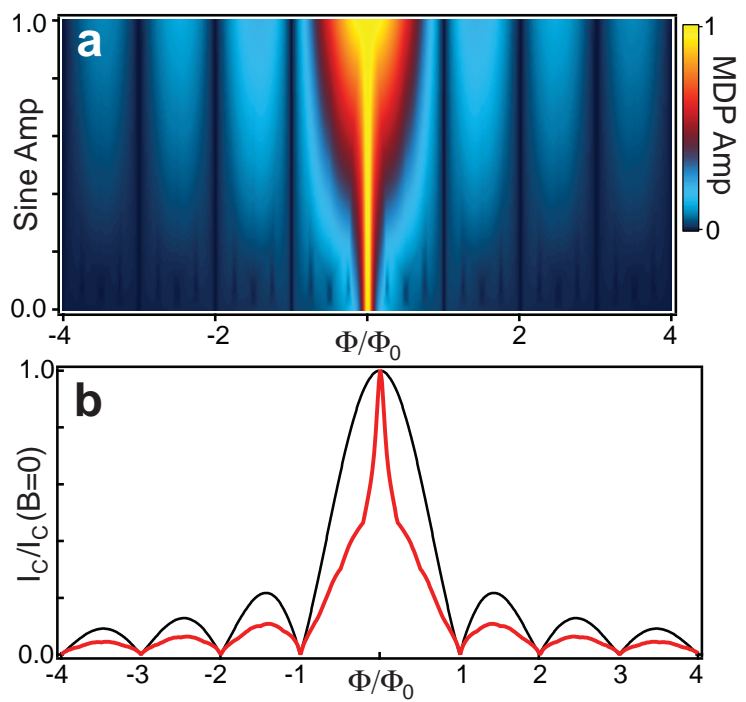

FIG. 9: Dependence of the MDP on the bulk contribution. (a) A 2D plot of the MDP as a function of $\Phi$ and the amplitude of the sinusoidal bulk contribution (Sine Amp). Sub- $\Phi_{0}$ minima are observed for values of Sine Amp less than 0.4. (b) A $1 \mathrm{D}$ cut in a at a value of Sine $A m p=0.5$ (red), showing a narrowed central lobe [compared to the Fraunhofer pattern (black)] in the MDP but no minima below $\Phi_{0}$.

to the one calculated in Fig. S5b is seen in Fig. 2b of Ref. [S20].

\section{A Graphite Control Device}

To confirm that the anomalous features observed in TI JJs are a result of the interface between a TI and a conventional superconductor, a JJ was created using $75 \mathrm{~nm}$-thick graphite as the weak link. The details of the device fabrication are identical to the fabrication of TI JJs, except the Ar ion mill step was not needed to produce low contact resistance. Instead, a 5 min UV-ozone exposure, typical in the creation of graphene devices ${ }^{S 21}$, was used. The results, taken at $T_{f}=12 \mathrm{mK}$, are shown in Fig. S5 where it is seen that the value of $I_{C} R_{N}$ is much higher at $244 \mu \mathrm{V}$ (Fig. S5a) and $B_{C}$ is $\sim 13 \mathrm{mT}$, closer to the predicted value of $14.3 \mathrm{mT}$ (black line) for a device of $(L, W)=(0.05,1) \mu \mathrm{m}$. The control device allows for the exclusion of two factors that might have caused the anomalous results in TI JJs. In Fig. S5a, a plot of $V$ vs. $I$ shows a value for the critical current of $8.1 \mu \mathrm{A}$ on a device of resistance $30.2 \Omega$. The larger value of $I_{C} R_{N}$ obtained for the control device rules out the possibility of the thermal effects caused by poor filtering being a source of the reduced $I_{C} R_{N}$ in the TI JJs. In Fig. S5b, $I_{C}$ vs. $B$ (red), extracted from a plot of $d V / d I(B, I)$ (inset of S5b) shows a more conventional dependence on $B$, closely matching the expected pattern (black). The high value of $B_{C}$ in the control device rules out the possibility of flux focusing ${ }^{S 22}$ reducing the values of $B_{C}$ in TI JJs.
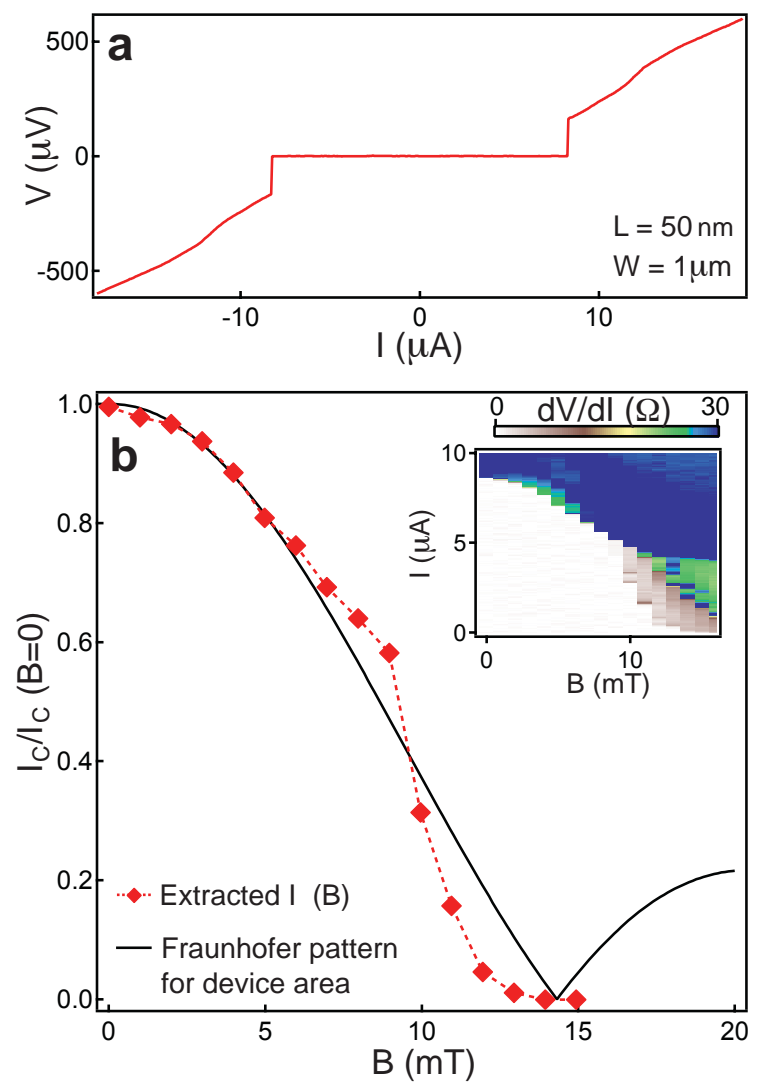

FIG. 10: Superconducting properties of a graphite control sample. (a) $V$ vs. $I$ for the $(L, W)=(50 \mathrm{~nm}, 1 \mu \mathrm{m})$ control device created from a $75 \mathrm{~nm}$-thick graphite weak link, producing a $I_{C} R_{N}$ of $244 \mu \mathrm{V}$, larger than the observed in TI JJs. (b) Extracted $I_{C}$ vs. $B$ (red) from $d V / d I$ (inset) giving a value of $B_{C}$ of $13 \mathrm{mT}$, close to the value of $14.3 \mathrm{mT}$ expected from the device area.

\section{Supporting References}

S1. Geim, A. K. and Novoselov, K. S., The rise of graphene. Nature Mater. 6, 183-191 (2007).

S2. Potok, R. M., Probing Many Body Effects in Semiconductor Nanostructures. Thesis, Harvard Univesity (2006).

S3. Analytis, J. G., et al., Two-dimensional surface state in the quantum limit of a topological insulator. Nature Phys. 6, 960-964 (2010).

S4. Analytis, J. G., et al., Bulk fermi surface coexistence with Dirac surface state in $\mathrm{Bi}_{2} \mathrm{Se}_{3}$ : A comparison of photoemission and Shubnikov de Haas measurements. Phys. Rev. B 81, 205407 (2010).

S5. Tinkham, M., Introduction to Superconductivity. Dover Publications, Mineola, New York (1996).

S6. Likharev, K. K., Superconducting weak links. Rev. Mod. Phys. 51, 101-159 (1979). 
S7. Qu, D.-X. et al., Quantum Oscillations and Hall Anomaly of Surface States in the Topological Insulator $\mathrm{Bi}_{2} \mathrm{Te}_{3}$, Science 329, 821 (2010).

S8. Likharev, K. K., Dynamics of Josephson Junctions and Circuits. Gordon and Breach Science Publishers, Amsterdam (1986).

S9. Blonder, G. E., Tinkham, M., and Klapwijk, T. M., Transition from metallic to tunneling regimes in superconducting microconstrictions: Excess current, charge imbalance, and supercurrent conversion. Phys. Rev. B 25, 4515-4532 (1982).

S10. Sacépé, B., et al., Gate-tuned normal and superconducting transport at the surface of a topological insulator. Nature Comm. 2 575, (2011).

S11. Furusaki, A., Josephson current carried by Andreev levels in sup conducting quantum point contacts. Superlat. and Microstruct. 25, 809-819 (1999).

S12. Kwon, H.-J., Sengupta, K., and Yakovenko, V. M., Fractional ac Josephson effect in p- and d-wave superconductors. Euro. Phys. J. B 37, 349-361 (2003).

S13. Fu, L. and Kane, C. L., Josephson current and noise at a superconductor/quantum-spin-Hallinsulator/superconductor junction. Phys. Rev. B 79, 161408 (2009).

S14. Kane, C. L. and Fisher, M. P. A. Fisher, Transport in a one-channel Luttinger liquid. Phys. Rev. Lett. 68, 1220-1223 (1992).

S15. Fu, L. and Kane, C. L., Superconducting proximity effect and majorana fermions at the surface of a topological insulator. Phys. Rev. Lett. 100, 096407 (2008).

S16. Ioselevich, P. A. and Feigel'man, M. V. Anomalous Josephson Current via Majorana Bound States in Topological Insulators. Phys. Rev. Lett. 106, 077003 (2011).

S17. Law, K. T. and Lee, P. A., Robustness of Majorana fermion induced fractional Josephson effect in multichannel superconducting wires. Phys. Rev. B 84, 081304 (2011).

S18. Barone, A. and Paternò, G. Physics and Applications of the Josephson Effect. Wiley-Interscience Publications, Canada (1982).

S19. Golubov, A. A., Kupriyanov, M. Y., and Il'ichev, E., The current-phase relation in Josephson junctions. Rev. Mod. Phys. 76, 411-469 (2004).

S20. Qu, F., et al., Strong superconducting proximity effect in $\mathrm{Pb}-\mathrm{Bi}_{2} \mathrm{Se}_{3}$ Hybrid Structures. arXiv:112.1683.

S21. Williams, J. R., Dicarlo, L. and Marcus, C. M. Quantum Hall Effect in a Gate-Controlled p-n Junction of Graphene. Science 317, 638-641 (2007).

S22. Ketchen, M. B., Gallagher, W. J., Kleinsasser, A. W., Murphy, S., and Clem, J. R., Proceedings of SQUID 85, p. 865, Berlin, (1985), edited by Hahlbahm, H. D. and Lubbig, H. 